\title{
A Novel Approach for Optimal Allocation and Sizing of Distributed Energy Storage System in Smart Grid
}

\author{
Menaka.K ${ }^{1}$, Mrs.S.Devil ${ }^{2}$ \\ ${ }^{I}$ (Dept of EEE, K.S.Rangasamy College of Technology,India), \\ ${ }^{2}$ (Associateprofessor \&, Dept of EEE, K.S.Rangasamy College of Technology, India)
}

\begin{abstract}
Distributed Energy Storage (DES) systems are a potential alternative to balance any instantaneous mismatch between supply and demand in the Smart Grid (SG). Optimization of location and sizing of DES uses analytical method which reduces the gain energy loss but it does not consider the cost. In this work, the medium voltage SG including Distributed Generation (DG) unit along with the DES system is taken. Controller based on Neural Network is used as solution procedure for the problem in optimal sizing and location of DES. The proposed work intends to offer a useful tool for analyzing potential advantage of distributed energy storage in $S G$ for both different possible regulatory schemes and services that are provided. A new cost based optimization strategy for optimal placement, sizing and control is the expected result and is implemented through MATLAB/Simulink model.
\end{abstract}

Keywords: Smart Grid, Distributed Energy Storage, Distributed Generation, Neural Networks, Renewable Energy Sources.

\section{Introduction}

In traditional power systems, large power generation plants located at adequate geographical places produce most of the power, which is then transferred toward large consumption centers over long distance transmission lines. The system control centers monitor and control the system continuously to ensure the quality of the power, namely the frequency and the voltage. However, the power system is changing, a large number of dispersed generation (DG) units, including both renewable and non-renewable sources such as wind turbines, wave generators, photovoltaic (PV) generators, small hydro, fuel cells and gas/steam powered combined heat and power (CHP) stations, are being developed . A wide spread use of renewable energy sources in distribution networks and a high penetration level will be seen in the near future. E.g., Denmark has a high penetration of wind energy in major areas of the country and today $14 \%$ of the whole electrical energy consumption is covered by wind energy. The main advantages of using renewable sources are the elimination of harmful emissions and the inexhaustible resources of the primary energy. However, the main disadvantage, apart from the higher costs, e.g., photovoltaic, is the uncontrollability[1]. The availability of renewable energy sources has strong daily and seasonal patterns. But the power demand by the consumers could have a very different characteristic. Therefore, it would be difficult to operate a power system installed with only renewable generation units due to the characteristic differences and the high uncertainty of the availability of the renewable sources. The way of fully exploiting the renewable energy is the grid connection, normally at distribution level. In this framework, electrical energy storage systems may play a crucial role, not by replacing existing components of the electricity value chain, but rather allowing the existing ones to do their job better and cheaper[2].

In fact, electricity storage devices, located where utility distribution systems are approaching a capacity limit, can provide significant economic assessment. These benefits are associated with deferred or avoided distribution equipment upgrades that often involve a large increment in capacity such as the addition of a second transformer in a substation or refurbishment in a long line segment. Distributed energy storage (DES) might be viewed both as a consumer and producer of power, thereby participating in the market as both a load and generator. Alternatively, storage might be viewed as an integral part of the distribution network, thereby removing it from the normal energy market. This might be linked to the question of who owns storage: load customers, generators, independent storage operators, or the network operator [6]. Regulation concerning the separation of roles in the electricity system varies from place to place and the ownership and operation of storage will vary as a consequence. Smart Grid (SG) is an electricity transmission and distribution network that has the ability to simplify and understand large amount of information and use it correctly, by making intensive use of automation, information and communication technologies. SG's are recently available in European countries, United States. Inorder to rectify the social problem of unbalance between supply and demand, Distributed Energy storage system is used.

Distributed Energy Storageis a local storage which is directly coupled to the grid along with the Distributed Generation (DG) and is a small scale electricity generation. DES systems such as super conducting energy storage, super capacitor energy storage and flywheel energy storage is a potential alternative inorder to 
balance any instantaneous mismatch between supply and demand in the SG [7]. Renewable Energy Sources(RESs) such as solar, wind connected to the grid is becoming important form of DG. Intermittency of generation from RES is a serious challenge at the distribution and at the transmission level which requires new protection and control strategies [5]. An electrical energy storage system plays a key role and is of great importance in the development of SG. Optimization of location and sizing of DES uses analytical method which reduces the gain energy loss but it does not consider the cost [4].

In this work, the medium voltage SG including DG unit along with the DES system is taken, which will avoid DG dispatching or curtailment as well as reducing the need of VAR generation from both RESs and $\mathrm{HV}$ grid. Neural network controller is used as solution procedure for the problem in optimal sizing and location of distributed energy storage system [3][8]. The proposed work intends to offer a useful tool for analyzing potential advantage of distributed energy storage in SG for both different possible regulatory schemes and services that are provided. A new cost based optimization strategy for optimal placement, sizing and control is implemented. For this optimization problem, MATLAB/Simulink model is used.

\section{Multi-Layer Neural Networks}

Eventually, despite the apprehensions of earlier workers, a powerful algorithm for apportioning error responsibility through a multi-layer network was formulated in the form of the backpropagation algorithm. The back propagation algorithm employs the Delta Rule, calculating error at output units, while error at neurons in the layer directly preceding the output layer is a function of the errors on all units that use its output. The effects of error in the output nodes are propagated backward through the network after each training case. The essential idea of back propagation is to combine a non-linear multi-layer perceptron-like system capable of making decisions with the objective error function of the Delta Rule.

\subsection{Network terminology}

A multi-layer feedforward backpropagation neural network is composed of

- an input layer of nodes,

- one or more intermediate layers of nodes, and

- $\quad$ an output layer of nodes

The output layer can consist of one or more nodes, depending on the problem at hand. In most classification applications, there will either be a single output node, or the same number of nodes in the output layer as there is classes. It is important to recognize that the term "multi-layer" is often used to refer to multiple layers of weights. This contrasts with the usual meaning of "layer", which refers to a row of nodes. For clarity, it is often best to describe a particular network by its number of layers, and the number of nodes in each layer.

\subsection{Back propagation algorithm}

In the employment of the back propagation algorithm, each iteration of training involves the following steps:

- A particular case of training data is fed through the network in a forward direction, producing results at the output layer.

- Error is calculated at the output nodes based on known target information, and the necessary changes to the weights that lead into the output layer are determined based upon this error calculation.

- The changes to the weights that lead to the preceding network layers are determined as a function of the properties of the neurons to which they directly connect until all necessary weight changes are calculated for the entire network.

The calculated weight changes are then implemented throughout the network, the next iteration begins, and the entire procedure is repeated using the next training pattern. In the case of a neural network with hidden layers, the backpropagation algorithm is given by the following three equations, where it is the "emitting" or "preceding" layer of nodes, $\mathrm{j}$ is the "receiving" or "subsequent" layer of nodes, $\mathrm{k}$ is the layer of nodes that follows $j$, ij is the layer of weights between node layers $i$ and $j, j k$ is the layer of weights between node layers $j$ and $\mathrm{k}$, weights are specified by $\mathrm{w}$, node activations are specified by a, delta values for nodes are specified by $\mathrm{d}$, subscripts refer to particular layers of nodes (i, j, k) or weights (ij, jk), "sub-subscripts" refer to individual weights and nodes in their respective layers, and epsilon is the learning rate:

where, if output node

$$
\Delta w_{i j_{m}}=\varepsilon \delta_{j_{p}} \alpha_{i_{q}}
$$

where, if intermediate node

$$
\delta_{j_{p}}=\alpha_{j_{p}}\left(1-\alpha_{j_{p}}\right)\left(t_{j_{p}}-\alpha_{j_{q}}\right)
$$

$$
\delta_{j_{p}}=\alpha_{j_{p}}\left(1-\alpha_{j_{p}}\right) \sum_{x=0}^{n} \delta_{k_{x}} w_{j k_{x}}
$$


Being based on the generalized Delta Rule, Equation (1a) states that the change in a given weight $\mathrm{m}$ located between layers $\mathrm{i}$ and $\mathrm{j}$ is equal to the products of:

- the learning rate

- the delta value for node $\mathrm{p}$ in layer $\mathrm{j}$

- the activation of node $q$ in layer $i$.

In practice, the learning rate is typically given a value of 0.1 or less; higher values may provide faster convergence on a solution, but may also increase instability and may lead to a failure to converge. The delta value for node $\mathrm{p}$ in layer $\mathrm{j}$ in Equation (1a) is given either by Equation (1b) or by Equation (1c), depending on the whether or not the node is in an output or intermediate layer. Equation (1b) gives the delta value for node $p$ of layer $\mathrm{j}$ if node $\mathrm{p}$ is an output node. Sigma activation function is used here instead of a simple linear activation function. Both sets of equations were determined by finding the derivative of the respective error functions with respect to any particular weight. Equation (1c) gives the delta value for node $p$ of layer $j$ if node $p$ is an intermediate node. This equation states that the delta value of a given node of interest is a function of the activation at that node (aj sub p), as well as the sum of the products of the delta values of relevant nodes in the subsequent layer with the weights associated with the vectors that connect the nodes.

\section{NETWORK PLANNING}

\subsection{Block diagram}

The block diagram of the work is shown in the Fig 1. Various types of power plants and its rating in KVA are given as the input to the controller based on neural network. Presently stored energy of the power plant in KVA, load demand at the particular time are also given as input to the controller. Usually the output if the neural network will be either 1 or 0 .

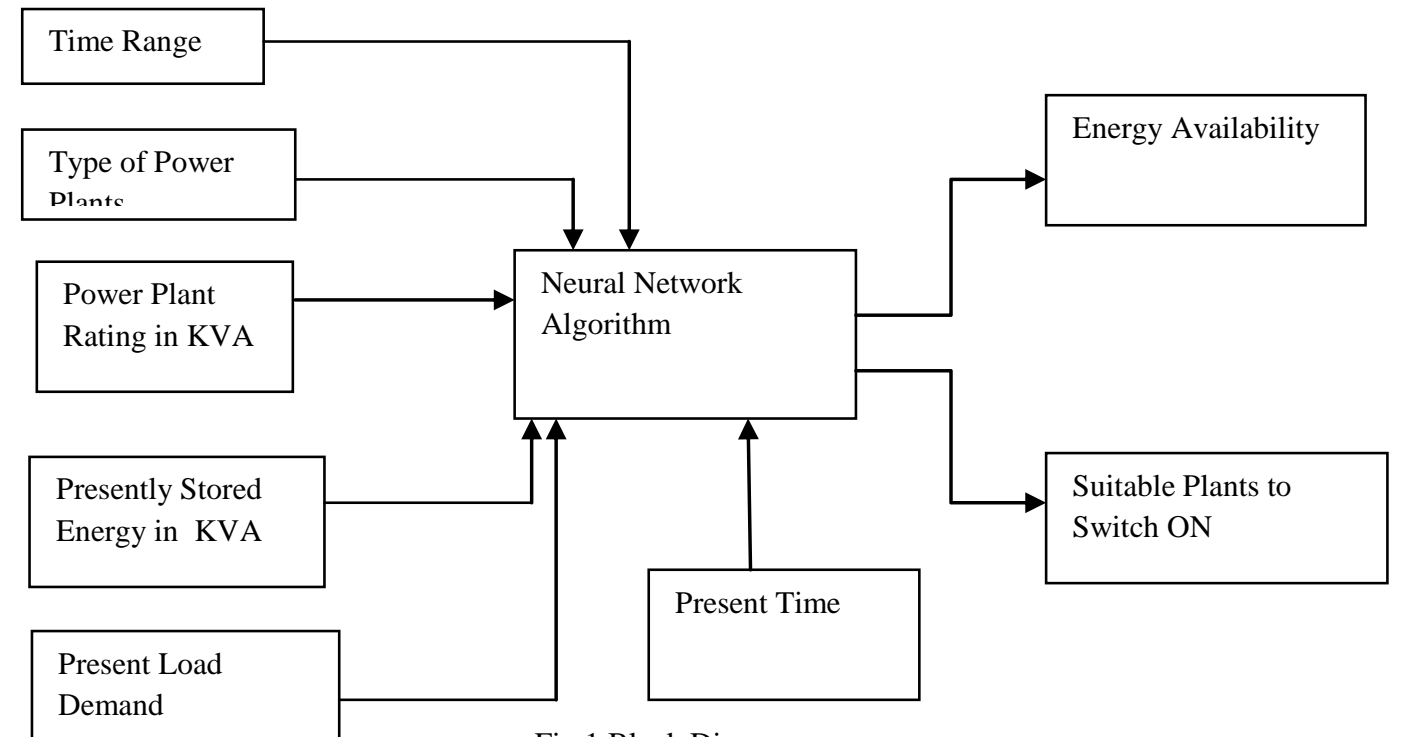

Fig.1 Block Diagram

Depending upon the load demand at that particular time, the neural network algorithm selects the type of power plant which satisfies the demand. It checks whether the selected power plants energy convince the load demand. Then it switch $\mathrm{ON}$ the suitable plants to operate in order to satisfy the load demand.

\subsection{Algorithm}

The algorithm for the controller based on the neural network for optimal allocation and sizing of distributed energy storage system in smart grid is as follows:

STEP 1: Collect the data for power generation and load demand of different power plants.

STEP 2: Create the neural network and initialize the weights and bias.

STEP 3: Train the neural network and load the test data into the network.

STEP 4: Check for the load demand set demand to 100

STEP 5: If load demand can be satisfied by switching on any one power plant then goto step 6 else goto step 7. STEP 6: If op is less than 100 then single power plant can be switched on the basis of $\operatorname{comb}(\mathrm{i},:)=[\operatorname{op} \operatorname{test}(1,3)$ test $(\mathrm{i}, 2)]$.

STEP 7: If it is greater than 100 then series of power plants are switched on to satisfy the demand.

STEP 8: Stop the process 


\subsection{Flowchart}

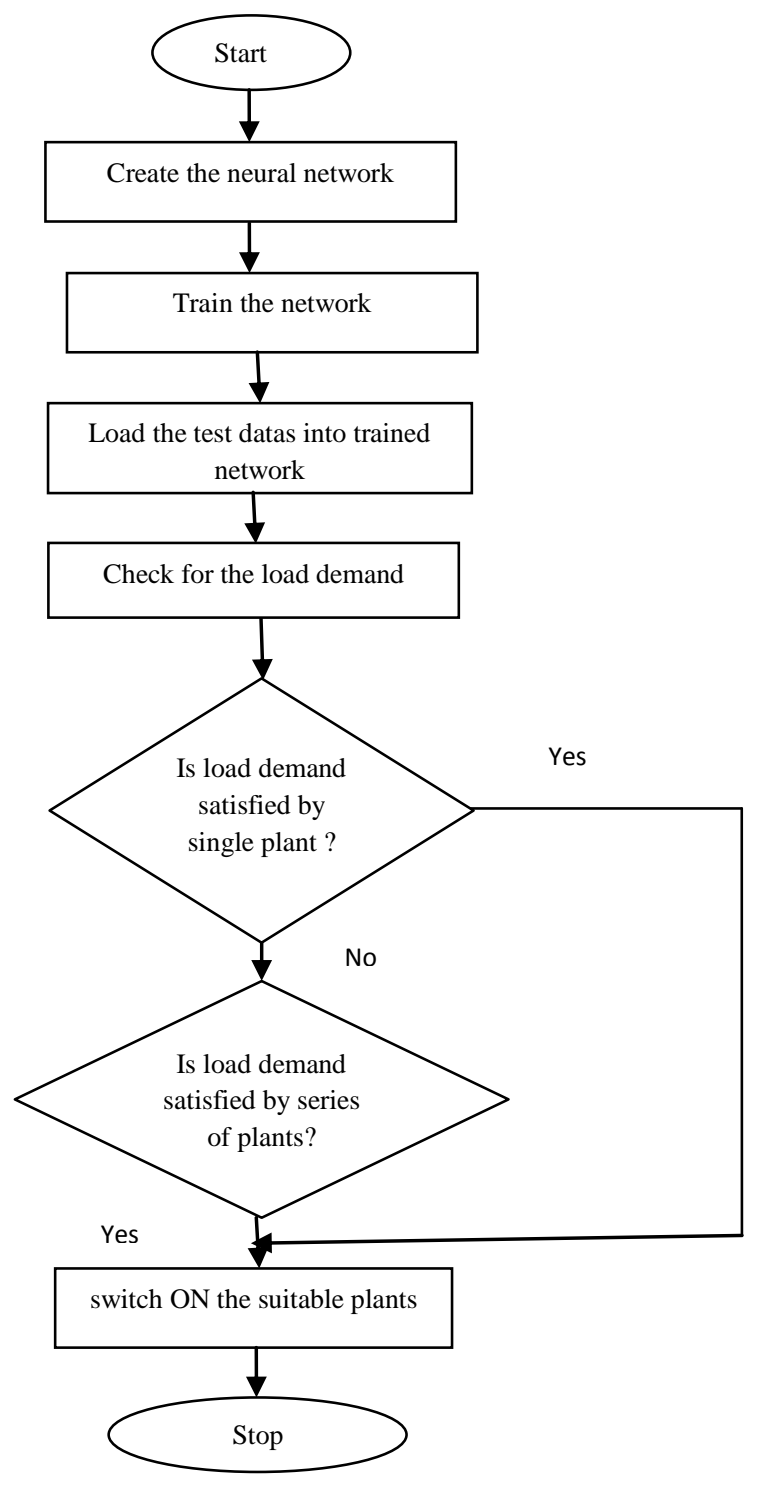

Fig. 2 Flowchart for proposed work

\section{Results And Discussion}

\subsection{Overview of the Training Process}

Two important steps in the application of neural networks for any purpose are training and testing. The first of the two steps namely training the neural network is discussed in this section. Training is the process by which the neural network learns from the inputs and updates its weights accordingly.

The artificial neural network model is built using a multilayer neural network shown in Fig.3. The system has three layers. The model is developed with one input layer, one output layer and hidden layer with 9 neurons. The network weights are initialized by using random valuesi.e.,0 and 1.

\section{Layer 1(Input Layer):}

The types of power plants, power plant rating, stored energy, load demand etc., are given as a input to the layer 1. The number of neurons of the input layer is based on the number of inputs of the problem. It is passed to the hidden layer.

\section{Layer 2(Hidden Layer):}

In feed forward, every input unit receives an input signal and sends the signal to the hidden units. Each hidden units then calculates the activation function and send its signal to the output layer. 


\section{Layer 3(Output Layer):}

Each output unit computes its activation to form the response of the network for the given input pattern. While training, each output unit compares its computed activation with its target value to determine the error associated with the pattern with that unit. Here the performance function used is mean squared error.

The Neural Network slowly learns the training set and slowly develops an ability to generalize upon this data and will eventually be able to produce an output when a new data is provided to it. During the training process, the neural network's weights are updated with the prime goal of minimizing the performance function. Fig.4 shows the MSE performance plot whose best training performance is 9.9555e-09 at epoch 195.

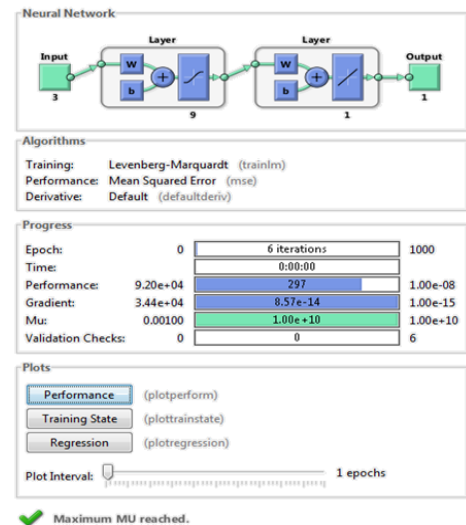

Fig. 3 Neural Network Model

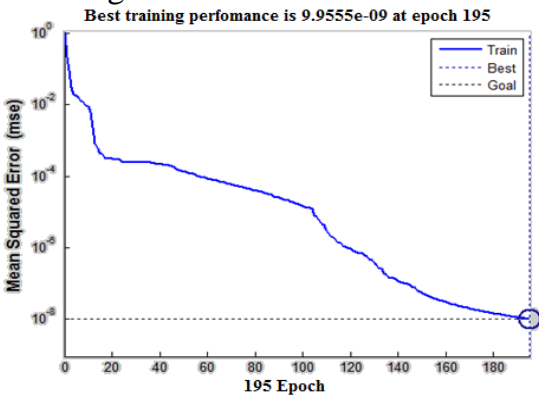

Fig. 4 MSE Performance

Fig.5 shows the plot for neural network Training regression. This graph is plotted for the target and the output. It checks whether the target and output of the neural network fit to each other.

\subsection{Overview of the Testing Process}

As mentioned already in the previous section, the next important step to be performed before the application of neural network is to test the trained network. Testing the artificial neural network is very important in order to make sure the trained network can generalize well and produce desired outputs when new data is presented to it.

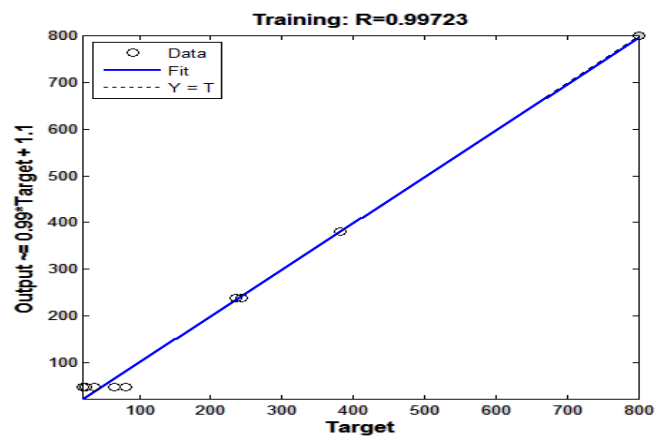

Fig.5 Regression Plot

The neural network toolbox in simulink by the math works divides the entire set of data provided to it into three different sets namely the training set, validation set and testing set. The training data set as indicated above is used to train the network by computing the gradient and updating the network weights. Fig.6 shows the graph for neural network training state up to 196 Epochs. 


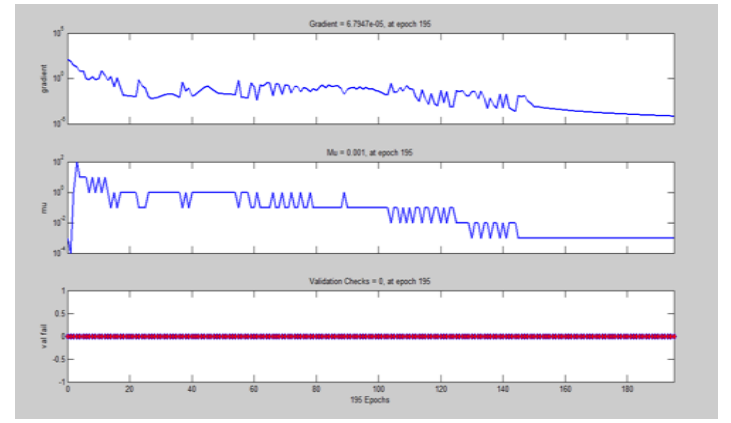

Fig.6 Validation Check

The validation set is provided during to the network during the training process and the error in validation data set is monitored throughout the training process. When the network starts over fitting the data, the validation errors increase and when the number of validation fails to increase beyond a particular value, the training process stops to avoid further over fitting the data and the network is returned at the minimum number of validation errors. The test set is not used during the training process but is used to test the performance of the trained network. If the test set reaches the minimum value of MSE at a significantly different iteration than the validation set, then the neural network will not be able to provide satisfactory performance.

\subsection{Simulation Result}

The simulation is carried out with the details of real time data of power generation in India. The test system data are reported in Appendix. For the load demand of $1250 \mathrm{KVA}$, simulation is carried out and results are as follows.

\section{Current Demand}

1250

You can turn on any one of following power plant

Table1: Results for the load demand of $1250 \mathrm{KVA}$

\begin{tabular}{|c|c|c|}
\hline Plant_Id & MaxRating(KVA) & $\begin{array}{c}\text { Stored Power } \\
\text { (KVA) }\end{array}$ \\
\hline 77 & 2400 & 1920 \\
\hline
\end{tabular}

Here for the load demand of $1250 \mathrm{KVA}$, each single plant can satisfy the demand from the given test data. Plant_ID 77 can satisfy the load individually. For the load demand of 2350 KVA, simulation is carried out and results are as follows.

Current Demand

2350

You can turn on following power plant in order to satisfy the load demand of 2800KVA, series of power plants from the test data has to be operated.

Table2: Results for the load demand of $1250 \mathrm{KVA}$

\begin{tabular}{|c|c|c|}
\hline Plant_Id & MaxRating(KVA) & $\begin{array}{c}\text { Stored Power } \\
\text { (KVA) }\end{array}$ \\
\hline 1 & 40 & 32 \\
\hline 2 & 2 & 2 \\
\hline 3 & 10 & 8 \\
\hline 5 & 1 & 1 \\
\hline 6 & 10 & 8 \\
\hline 7 & 3 & 2 \\
\hline 8 & 2 & 2 \\
\hline 9 & 3 & 2 \\
\hline 10 & 25 & 20 \\
\hline 11 & 30 & 24 \\
\hline 12 & 1 & 1 \\
\hline 13 & 1 & 1 \\
\hline 14 & 5 & 4 \\
\hline 15 & 1 & 1 \\
\hline 16 & 3 & 2 \\
\hline 17 & 1 & 1 \\
\hline 18 & 1 & 1 \\
\hline 19 & 10 & 8 \\
\hline 20 & 50 & 40 \\
\hline 22 & 33 & 26 \\
\hline
\end{tabular}




\begin{tabular}{|c|c|c|}
\hline 23 & 30 & 24 \\
\hline 24 & 25 & 20 \\
\hline 25 & 22 & 18 \\
\hline 26 & 21 & 17 \\
\hline 27 & 20 & 16 \\
\hline 28 & 15 & 12 \\
\hline 29 & 15 & 12 \\
\hline 30 & 14 & 11 \\
\hline 31 & 14 & 11 \\
\hline 32 & 12 & 10 \\
\hline 33 & 11 & 9 \\
\hline 34 & 10 & 8 \\
\hline 35 & 10 & 8 \\
\hline 36 & 15 & 12 \\
\hline 37 & 11 & 9 \\
\hline 38 & 56 & 45 \\
\hline 39 & 10 & 8 \\
\hline 40 & 20 & 16 \\
\hline 41 & 54 & 53 \\
\hline 57 & 12 & 10 \\
\hline 61 & 55 & 44 \\
\hline 63 & 60 & 48 \\
\hline 70 & 60 & 48 \\
\hline 67 & 1500 & 1200 \\
\hline 66 & 965 & 772 \\
\hline
\end{tabular}

Available

2617

\section{Conclusion}

The wide deployment of intermittent solar and wind generation is challenging transmission and distribution systems. DER management, energy storage, dispatch of wind and solar resources as well as demand response strategies could alleviate some of these challenges. Among those remedies, the integration of DES systems may exalt the potentialities of SGs to make the SGO an aggregator capable to offer also ancillary services to TSO, without tasking to RES excessive participation to voltage regulation. The proposed approach is a useful tool for analyzing potential advantages of distributed energy storages in SGs with reference todifferentpossibleconceivableregulatoryschemesandservicestobeprovided. Thus the neural network has been trained and the power plants have been switched ON depending upon the demand condition. To meet the required demand, first single suitable power plants have been ON. If it does not meet out the demand the combination of units in the power plants are switched ON to meet out the demand. Thus the results have been discussed already.

\section{References}

[1] AlaaMohd, EgonOrtjohann, Andreas Schmelter, NedzadHamsic, Danny Morton, "Challenges in Integrating Distributed Energy Storage Systems into Future Smart Grid", IEEE Transaction, 2008.

[2] AnvariMoghaddam.A, A.R. Seifi, "Study of forecasting renewable energies in smart grids using linear predictive filters and neural networks", IET Renewable Power Generation., Vol. 5, Iss. 6, pp. 470-480, April 2011.

[3] Benjamin Kroposki,P.K. Sen, Keith Malmedal, "Optimum Sizing and Placement of Distributed and Renewable Energy Sources in Electric Power Distribution Systems", IEEE Transaction, 2013.

[4] G.Celli, S. Mocci, F. Pilo, and M. Loddo "Optimal Integration Of Energy Storage In Distributed Networks", IEEE Bucharest Power Tech Conference, 2009.

[5] FredeBlaabjerg, Zhe Chen, SoerenBaekhoejKjaer, "Power Electronics as Efficient Interface in Dispersed Power Generation Systems", IEEE Transactions On Power Electronics, Vol. 19, No. 5, September 2004.

[6] ImanZiari, AlirezaJalilian, "A New Approach for Allocation and Sizing of Multiple Active Power-Line Conditioners", IEEE Transactions On Power Delivery, Vol. 25, No. 2, April 2010.

[7] Marcelo G. Molina, "Distributed Energy Storage Systems for Applications in Future Smart Grids", IEEETransaction, 2012.

[8] Umar Farooq, Muhammad Amar, EitzazulHaq, Muhammad UsmanAsad, Hafiz Muhammad Atiq, "Microcontroller Based Neural Network Controlled Low Cost Autonomous Vehicle”, IEEE Computer society, 2010.

[9] http://en.wikipedia.org/wiki/Wind power in India

[10] http://en.wikipedia.org/wiki/Renewable energy in India

APPENDIX

Table 3 : Test System Data

\begin{tabular}{|l|c|c|c|}
\hline Name of Power Plants & DC Peak Power & $\mathbf{8 0 \%}$ Load & Plant ID \\
\hline Adani Power Bitta,Gujarat & 40 & 32 & PP001 \\
\hline Azure Power - Photovoltaic Plant & 2 & 2 & PP002 \\
\hline Azure Power - Sabarkantha, Gujarat & 10 & 8 & PP003 \\
\hline Charanka Solar Park - Charanka, Gujarat & 214 & 171 & PP004 \\
\hline Gandhinagar Solar Plant & 1 & 1 & PP005 \\
\hline
\end{tabular}




\begin{tabular}{|c|c|c|c|}
\hline $\begin{array}{l}\text { Green Infra Solar Energy Limited- Rajkot, } \\
\text { Gujarat }\end{array}$ & 10 & 8 & PP006 \\
\hline Itnal Photovoltaic Plant, Belgaum & 3 & 2 & PP007 \\
\hline Jamuria Photovoltaic Plant & 2 & 2 & PP008 \\
\hline Kolar Photovoltaic Plant & 3 & 2 & PP009 \\
\hline $\begin{array}{l}\text { Mithapur Solar Power Plant - Mithapur, } \\
\text { Gujarat (Tata Power) }\end{array}$ & 25 & 20 & PP010 \\
\hline $\begin{array}{l}\text { Moser Baer Clean Energy Limited - } \\
\text { Banaskantha, Gujarat }\end{array}$ & 30 & 24 & PP011 \\
\hline NDPC Photovoltaic Plant & 1 & 1 & PP012 \\
\hline REHPL - Sadeipali, (Bolangir) Orissa & 1 & 1 & PP013 \\
\hline Sivaganga Photovoltaic Plant & 5 & 4 & PP014 \\
\hline Tata - Mayiladuthurai, Tamil Nadu & 1 & 1 & PP015 \\
\hline Tata - Mulshi, Maharashtra & 3 & 2 & PP016 \\
\hline TATA - Osmanabad, Maharastra & 1 & 1 & PP017 \\
\hline Thyagaraj stadium Plant-Delhi & 1 & 1 & PP018 \\
\hline $\begin{array}{l}\text { Waa Solar Power Plant - Surendranagar, } \\
\text { Gujarat (Madhav Power) }\end{array}$ & 10 & 8 & PP019 \\
\hline $\begin{array}{l}\text { Welspun Energy 50MW Rajasthan Solar } \\
\text { Project - Phalodhi, Rajasthan }\end{array}$ & 50 & 40 & PP020 \\
\hline Vankusawade Wind Park & 259 & 207 & PP021 \\
\hline Cape Comorin & 33 & 26 & PP022 \\
\hline Kayathar Subhash & 30 & 24 & PP023 \\
\hline Ramakkalmedu & 25 & 20 & PP024 \\
\hline Muppandal Wind & 22 & 18 & PP025 \\
\hline Gudimangalam & 21 & 17 & PP026 \\
\hline Puthlur RCI & 20 & 16 & PP027 \\
\hline Lamda Danida & 15 & 12 & PP028 \\
\hline Chennai Mohan & 15 & 12 & PP029 \\
\hline Jamgudrani MP & 14 & 11 & PP030 \\
\hline Jogmatti BSES & 14 & 11 & PP031 \\
\hline Perungudi Newam & 12 & 10 & PP032 \\
\hline Kethanur Wind Farm & 11 & 9 & PP033 \\
\hline Hyderabad APSRTC & 10 & 8 & PP034 \\
\hline Muppandal Madras & 10 & 8 & PP035 \\
\hline Shah Gajendragarh & 15 & 12 & PP036 \\
\hline Shah Gajendragarh & 11 & 9 & PP037 \\
\hline Acciona Tuppadahalli & 56 & 45 & PP038 \\
\hline Poolavadi Chettinad & 10 & 8 & PP039 \\
\hline Shalivahana Wind & 20 & 16 & PP040 \\
\hline Dangiri Wind Farm & 54 & 43 & PP041 \\
\hline Baira Suil & 180 & 144 & PP042 \\
\hline Balimela power Station & 510 & 408 & PP043 \\
\hline Bansagar Dam & 425 & 340 & PP044 \\
\hline Bargi Dam & 105 & 84 & PP045 \\
\hline Baspa-II & 300 & 240 & PP046 \\
\hline Bhakra Dam & 1325 & 1060 & PP047 \\
\hline Chamera-I & 540 & 432 & PP048 \\
\hline Chamera-II & 300 & 240 & PP049 \\
\hline Dehar (Pandoh) & 990 & 792 & PP050 \\
\hline Dhauliganga-I & 280 & 224 & PP051 \\
\hline Dulhasti & 390 & 312 & PP052 \\
\hline Ghatghar Pumped Storage Scheme & 250 & 200 & PP053 \\
\hline Hirakud Power System,Burla \& Chipilima & 348 & 278 & PP054 \\
\hline Idukki & 780 & 624 & PP055 \\
\hline Indira Sagar & 1000 & 800 & PP056 \\
\hline Jayakwadi Dam & 12 & 10 & PP057 \\
\hline Kalinadi & 1240 & 992 & PP058 \\
\hline Karcham Wangtoo & 1000 & 800 & PP059 \\
\hline Koyna & 1960 & 1568 & PP060 \\
\hline Linganamakki Dam & 55 & 44 & PP061 \\
\hline Loktak & 105 & 84 & PP062 \\
\hline Madikheda Dam & 60 & 48 & PP063 \\
\hline Mettur Dam & 240 & 192 & PP064 \\
\hline Mulshi Dam & 150 & 120 & PP065 \\
\hline Nagarjunasagar & 965 & 772 & PP066 \\
\hline Nathpa Jhakri & 1500 & 1200 & PP067 \\
\hline Omkareshwar & 520 & 416 & PP068 \\
\hline Pong & 396 & 317 & PP069 \\
\hline Rangeet & 60 & 48 & PP070 \\
\hline Salal & 690 & 552 & PP071 \\
\hline
\end{tabular}


A Novel Approach for Optimal Allocation and Sizing of Distributed Energy Storage System in Smart

\begin{tabular}{|l|c|c|c|}
\hline Sardar Sarovar & 1450 & 1160 & PP072 \\
\hline Sharavathi & 1469 & 1175 & PP073 \\
\hline Srisailam Dam & 1670 & 1336 & PP074 \\
\hline Tanakpur & 120 & 96 & PP075 \\
\hline Teesta-V & 510 & 408 & PP076 \\
\hline Tehri Dam & 2400 & 1920 & PP077 \\
\hline Upper Indravati & 600 & 480 & PP078 \\
\hline Uri Hydroelectric Dam & 480 & 384 & PP079 \\
\hline
\end{tabular}

